\title{
OBJETOS DE APRENDIZAGEM E LOUSAS DIGITAIS INTERATIVAS: UMA PROPOSTA DE AVALIAÇÃO DE OBJETOS DE APRENDIZAGEM PARA ENSINO DE MATEMÁTICA
}

\author{
Silvio Henrique FISCARELLI ${ }^{1}$ \\ Camila Lourenço MORGADO ${ }^{2}$ \\ Monique Alves FÉLIX ${ }^{3}$
}

Resumo: As atuais condições da sala de aula tendem a torna o processo de ensinoaprendizagem unidirecional e expositivo, tendo um impacto negativo na aprendizagem, direcionando as ações para uma aprendizagem mecânica e não significativa. Uma das possibilidades para melhorar a qualidade do ensino é inovar as metodologias e variar as formas de apresentação de informações para nossos alunos, como o uso da tecnologia no processo de ensino. A Lousa Digital Interativa (LDI) está entre as tecnologias que vêm sendo implantadas nas escolas brasileiras. Uma das possibilidades promissoras para agregar valor ao uso das LDI durante as aulas são os "Objetos de Aprendizagem" (OA). Porém, um dos problemas é que muitas vezes os OA não são plenamente adequados à dinâmica da LDI, seja do ponto de vista funcional ou pedagógico. $\mathrm{O}$ objetivo deste trabalho é analisar e propor um conjunto de indicadores que avaliam os Objetos de Aprendizagem para serem utilizados em conjunto com Lousas Digitais Interativas. O levantamento e definição dos indicadores de avaliação foi realizada a partir da revisão da literatura sobre o tema e com base em experiências de uso de LDI em uma escola Municipal de Ensino Fundamental. Após a definição do conjunto de indicadores foi realizada a avaliação de uma amostra de 30 OA destinados ao ensino de Matemática na $3^{\text {a }}$ série do Ensino Fundamental. Os resultados da avaliação sinalizam que os indicadores propostos são adequados para uma pré-análise dos OA e auxiliam no processo de seleção dos mesmos.

Palavras-chave: Tecnologia. Objetos de aprendizagem. Lousas digitais. Avaliação de software. Indicadores.

\section{INTRODUÇÃO}

O baixo rendimento escolar brasileiro vem sendo alvo de diversas pesquisas que buscam expor quais os possíveis fatores responsáveis por esta situação. No entanto, as questões educacionais são complexas e as respostas sempre envolvem várias dimensões que compõem o processo educacional. Para compreender este fenômeno, e melhorar a

\footnotetext{
${ }^{1}$ Departamento de Didática. UNESP - Universidade Estadual Paulista “Júlio de Mesquita Filho". Faculdade de Ciências e Letras de Araraquara. Araraquara - SP - Brasil. 14800-901 silviohf@yahoo.com.

${ }^{2}$ Mestranda em Educação Escolar. UNESP - Universidade Estadual Paulista "Júlio de Mesquita Filho". Faculdade de Ciências e Letras. Araraquara - SP - Brasil. 14800-901 - mila.morgado08@ gmail.com.

${ }^{3}$ Graduanda em Pedagogia. UNESP - Universidade Estadual Paulista "Júlio de Mesquita Filho". Faculdade de Ciências e Letras. Araraquara - SP - Brasil. 14800-901 - moni.felix@ hotmail.com.
} 
qualidade do ensino, é necessário considerar aspectos como o investimento na educação, a formação do professor, o número alto de alunos por classe, a jornada extensiva do professor, as questões socioeconômicas do alunado e, principalmente, a dinâmica da aprendizagem em sala de aula.

As informações transmitidas nas escolas quase sempre estão submetidas ao contexto da sala de aula, afastando-se do contexto do conhecimento real (HENNING, 1998). As condições físicas, organizacionais e psicológicas da sala de aula tendem a torna o processo de ensino-aprendizagem unidirecional e expositivo, com ênfase nos recursos verbais (textos, lousa e fala do professor). Esta situação tem um impacto negativo na aprendizagem, direcionando as ações para uma aprendizagem mecânica e não significativa; ou seja, na impossibilidade de compreender quais processos e relações estão por trás de um determinado conhecimento, o aluno passa a "memorizar" como usar as fórmulas e equações, os conceitos mais utilizados no seu curso e os passos de procedimento, mas não sabem direito quando e por que os utilizam.

Várias pesquisas, avaliações e relatórios (PEDERSEN et al., 2006; BALANSKAT; BLAMIRE; KEFALA, 2006; BECTA, 2007; UNITED STATES OF AMERICA, 2010) têm apontado que uma das possibilidades para melhorar a qualidade do ensino é inovar as metodologias e variar as formas de apresentação de informações para nossos alunos. Essas inovações, em grande parte, estão associadas ao uso da tecnologia no processo de ensino.

Entre as tecnologias que estão adentrando a escola está a chamada Lousa Digital Interativa (LDI), que vem sendo implantada por diversos municípios em todo o Brasil e, principalmente, no Estado de São Paulo, atualmente, temos notícias que dezenas de municípios, entre eles: Araraquara, Itu, Mongaguá, Ribeirão Pires, Jaú, Jaguariúna, Elias Fausto, Salto, Alumínio, São Carlos, Limeira, São Caetano, que iniciaram o processo de implementação das lousas digitais, acreditando que este recurso possa melhorar todo processo de ensino e aprendizagem, de todas suas relações, e em toda sua dinâmica.

A LDI é um recurso tecnológico que possibilita o desenvolvimento de atividades didáticas multimídia, ou seja, o uso de imagens, textos, sons, vídeos, páginas da internet, dentre outras opções que estejam em formato digital. Tecnicamente a LDI compreende um conjunto de equipamentos tecnológicos organizados de forma a cumprir uma tarefa específica. Esses equipamentos são: um sistema de interação motora com os usuários; um projetor, para projetar as informações do computador; o 
computador, que gerencia todas as interações, e, o software da lousa digital interativa, que oferece diversas ferramentas, possibilitando que seus usuários prepararem atividades, apresentações e ações, conjuntamente aos demais aplicativos do computador.

Porém o Hardware é somente ponto de partida para a implementação das tecnologias na escola, o fundamental para que ela possa realmente melhorar a aprendizagem é dispor de recursos de aprendizagem adequados para trabalhar o currículo, bem como ter professores formados para trabalhar com esses equipamentos e os conteúdos no formato digital. Em uma pesquisa desenvolvida por Bosley e Moon (2003), concluiu que a ausência de softwares pode dispersar os alunos do processo de aprendizagem. Os autores também comentam sobre outros fatores relacionados aos softwares que inibem o uso das TIC, como o alto custo das licenças de software, e também a falta de tempo disponível para os professores testarem e aprenderem utilizar os softwares adequadamente.

Uma das possibilidades promissoras para agregar valor ao uso das LDI durante as aulas são os "Objetos de Aprendizagem" (OA), que consistem em recursos digitais, tais como vídeos, imagens, áudios, textos, gráficos, tabelas, mapas, jogos, simulações, animações e infográficos que podem ser utilizados isoladamente ou agrupados em um único aplicativo.

No entanto, um dos grandes problemas deste recurso é que muitas vezes os OA não são plenamente adequados à dinâmica da LDI, seja do ponto de vista funcional ou pedagógico, cabendo ao professor analisar e avaliar as características que consideram indispensáveis e que revelam se aquele material é de qualidade ou não. Tratando-se de duas tecnologias que ainda estão sendo implantadas de forma assistemática no Brasil, não existem parâmetros ou indicadores que possibilitem uma avaliação consistente sobre a qualidade de OA para uso em Lousas Digitais Interativas.

A proposta de deste trabalho é analisar e propor um conjunto de indicadores que avaliam os Objetos de Aprendizagem para serem utilizados em conjunto com Lousas Digitais Interativas. Neste sentido foi realizada uma revisão da literatura sobre avaliação de OA e análise de uma experiência de uso de LDI em uma escola Municipal de Ensino Fundamental. Após a definição dos indicadores de avaliação vamos analisar uma amostra de $30 \mathrm{OA}$ destinados ao ensino de Matemática na $3^{\circ}$ série do Ensino Fundamental. Pretende-se, com este procedimento, analisar e verificar a adequação dos indicadores propostos. 


\section{Avaliação de Objetos de Aprendizagem e Lousas Digitais Interativas}

Para Kochanski (2009), o uso das novas tecnologias na educação se torna cada vez mais importante devido ao impacto que os avanços na área da computação causaram na sociedade atual. A geração de nativos digitais possui uma estrutura mental que possibilidade uma aprendizagem mais acelerada, que pode ser oferecida por meio de novas tecnologias e métodos diferenciados.

Segundo Kochanski (2009), o processo de aprendizagem se dá por meio da interação entre sujeito e meio ambiente, interação essa que oferece desafios, ativando estruturas mentais que permitem a elaboração de esquemas para sua adaptação ou transformação do ambiente. Por meio dos objetos de aprendizagem é possível desenvolver-se experiências que reduzem a lacuna entre teoria e prática, proporcionando assim um processo de aprender fazendo, utilizando elementos virtuais.

Para Almeida, Chaves e Coutinho (2010), a utilização dos objetos de aprendizagem oferece algumas vantagens como, sua facilidade de uso, baixo custo, melhora na qualidade de ensino, possibilidade de acompanhar os avanços dos alunos e conhecer suas dificuldades.

De acordo com Kochanski (2009), para que se possa utilizar os objetos de aprendizagem de maneira correta é preciso primeiramente que se defina quais níveis de domínio cognitivo se deseja atingir, desta forma é necessário preparar o conteúdo dos materiais os resultados de aprendizagem que se procura.

Reategui e Finco (2010) afirmam que o uso dos objetos de aprendizagem dentro do contexto escolar ainda perpassa por diversas dificuldades, entre elas está a falta de preparo por parte dos professores para utilizar essa nova tecnologia. Diante da imensa variedade de recursos que podem auxiliar a aprendizagem dos alunos, o professor muitas vezes se sente impotente, sem conhecer a qualidade dos materiais ou como selecioná-los.

Ainda de acordo com Reategui e Finco (2010), a escassez de materiais específicos é outro aspecto que dificulta o uso das novas tecnologias no processo de aprendizagem dos alunos. Uma alternativa para a pouca quantidade de materiais seria a produção desses objetos de aprendizagem por parte dos próprios professores, porém a complexidade dessa produção torna essa tarefa algo muito difícil. Os repositórios de universidades também contribuem para o acesso por parte dos professores a uma maior variedade de materiais, mas a falta de avaliação da qualidade desses objetos de aprendizagem reforça ainda mais a resistência ao seu uso. 
Os principais problemas apresentados pelos materiais são em relação a qualidade do conteúdo, podendo estar relacionado a uma abordagem superficial da temática, ausência de contextualização, falta do uso de exemplos, analogias e ludicidade (ALMEIDA; CHAVES; COUTINHO, 2010).

A preocupação com a avaliação de OA não é uma questão nova, vem sendo discutida desde a década de 90, vários autores, entre eles, Andrews e Goodson (1980), Campos e Rocha (1991), Oliveira (2001), Vieira (2004), se preocuparam em elaborar algumas diretrizes para a avaliação desses. No entanto, o crescimento dos repositórios e o grande acesso à tecnologia pelas escolas nos dias atuais intensifica a necessidade de discutir e refinar as propostas e modelos de avaliação para os OA.

Desta forma, pode-se concluir que existe a necessidade da criação de diretrizes para avaliar e selecionar os objetos de aprendizagem. Para que o material atenda às necessidades dos estudantes é preciso analisá-los pela perspectiva de sua funcionalidade, eficiência e confiabilidade. Outros aspectos que precisam ser considerados em sua avaliação são suas características pedagógicas e técnicas, buscando sempre alinhar os objetos de aprendizagem as práticas pedagógicas.

\section{Ensino de matemática no ensino fundamental}

Santos e Moita (2009) afirmam que, apesar dos avanços da tecnologia no suporte ao processo de ensino e aprendizagem, ainda é possível observar nas escolas que o ensinar e aprender matemática continua sendo uma tarefa, que gera medos e dificuldades por parte de professores e alunos. O processo de ensino e aprendizagem de matemática possui três variáveis fundamentais e relações que se estabelecem entre si, aluno, professor e conhecimento. O conhecimento matemático trata-se de uma ciência viva, que está aberto a integração de novos conhecimentos, para que o aluno possa apreender e generalizar tais apreensões de saberes.

Para Santos e Moita (2009) as dificuldades encontradas no ensino de matemática não estão concentradas apenas na complexidade para se aprender os conteúdos, mas também nas características cognitivas, sociais e culturais dos alunos, além da forma como serão ensinados.

De acordo com Santos e Moita (2009), contextualizar os conteúdos, para que estes não sejam ensinados como algo abstrato, que não faz parte do nosso cotidiano, contribui para que os alunos os tornem mais significativos, relacionando-os com suas vivencias, consequentemente atribuindo sentido a sua aprendizagem. 
Ainda de acordo com Santos e Moita (2009), os objetos de aprendizagem oferecem uma interface nova para o processo de ensino e aprendizagem de matemática, oferecendo simulações nas quais pode-se observar regularidades, generalizando e transferindo conhecimentos a outros contextos. Tal movimento transforma o conhecimento em algo pleno, pois permite que ele seja aplicado em outras situações que não sejam a de sua origem. Essas conexões são fundamentais para que para que os alunos possam compreender os conteúdos, contribuindo para o desenvolvimento de sua capacidade de resolução de problemas.

Segundo Castro-Filho et al. (2008), o uso dos objetos de aprendizagem no ensino de matemática oferece aos alunos a possibilidade de conexões entre diferentes formas de representação de conceitos, sejam essas representações intuitivas ou abstratas. Estabelecer conexões entre múltiplas formas de representações é apontado como um auxílio no desenvolvimento de conceitos matemáticos.

A seleção dos objetos de aprendizagem deve considerar diversos aspectos, partindo-se do planejamento da aula e do acompanhamento do professor. Para que este acompanhamento ocorra é preciso que o professor já conheça o objeto de aprendizagem, adequando seu uso as discussões realizadas em sala de aula, podendo assim antecipar questões ou dificuldades dos alunos em relação ao conteúdo trabalhado (CASTROFILHO et al., 2008).

\section{Uma proposta de Modelo de Avaliação para Objetos de Aprendizagem}

O principal objetivo deste artigo é apresentar e verificar a pertinência de um modelo de avaliação de Objetos de Aprendizagem para uso em Lousas Digitais Interativas, esse modelo é composto por um conjunto de diretrizes ou indicadores que podem ser empregados no processo de apreciação da adequação técnica e pedagógica dos mesmos. Trata-se de uma avaliação preliminar e não em circunstâncias reais de sala de aula, portanto tem como objetivo auxiliar no processo de seleção de OA apresentam potencial para atuarem como.

Tal proposta fundamenta-se na importância das escolas e educadores e pesquisadores da área de tecnologia disporem de um instrumento preliminar para reconhecer e avaliar características importantes nestes materiais, características que podem atestar ou não sua adequação e qualidade para o processo de ensinoaprendizagem. 
Um modelo pode ser definido como a estruturação e representações de um conceito teórico, de "[...] uma ideia, um objeto, um evento, um processo ou um sistema" (GILBERT; BOULTER; RUTHERFORD, 1998), de maneira sistematizada através do estabelecimento de relações.

Modelos são essenciais para a produção, difusão e aceitação do conhecimento científico (GIERE, 1988; GILBERT; BOULTER; RUTHERFORD, 1998; TOMASI, 1988). Embora sua natureza epistemológica esteja aberta ao debate, eles funcionam como uma ponte entre a teoria científica e o mundo como experiência ('realidade'). Eles podem ser representações simplificadas de uma realidade observada, produzida para fins específicos, para os quais as abstrações da teoria são, então, aplicadas.

O Quadro 1 apresenta o conjunto de indicadores propostos, dos quais os mais presentes e recomendados na literatura sobre avaliação do OA de maneira mais ampla, outros estão sendo sugeridos a partir de nossa experiência em pesquisa e uso de Lousas Digitais Interativas no Ensino Fundamental. Tais indicadores, de uma maneira geral, são a expressão das necessidades dos usuários (alunos/Professores), explicitadas em termos quantitativos ou qualitativos, e têm por objetivo definir a presença ou ausência das características desejadas (ROCHA; CAMPOS, 1993).

Para testar o modelo foi sorteada uma amostra aleatória de 30 objetos de aprendizagem de um montante de 120 objetos de Aprendizagem destinados ao ensino de Matemática, referente ao currículo do $3^{\circ}$ ano do Ensino Fundamental.

A partir do conjunto de critérios definidos no Quadro1 foi elaborado um instrumento de avaliação com características de checklist, ao qual cada um dos 30 objetos foi submetido. O checklist tem como objetivo averiguar cada um dos atributos desejados no OA em forma de perguntas objetivas. Cada pergunta contemplava somente um atributo e tem como alternativas 3 tipos de respostas: "S" (Sim) para as proposições verdadeiras; "N" (Não) para as proposições falsas; "NA" (Não Aplicável) para as proposições que fazem referência a uma característica que não se ajusta ao OA que está sendo avaliado.

O preenchimento do checklist para cada OA aconteceu fora da sala de aula, momento em que é realizada uma exploração geral das funcionalidades e interações didático-pedagógicas propiciadas pelo recurso. Um usuário com experiência sobre o OA e currículo identificou as potenciais evidências para cada um dos critérios desejados, indicando o quanto cada evidência fornecida atende ao resulta do esperado. 
Quadro1 - Lista de dimensões e indicadores para avaliação

\begin{tabular}{|c|c|}
\hline Dimensão & Indicadores \\
\hline \multirow[t]{10}{*}{ Design } & Visualmente atraente? \\
\hline & Tamanho adequado da tela? \\
\hline & Ajusta se adequadamente a tela? \\
\hline & Faz uso adequado do teclado? \\
\hline & Faz uso adequado do Mouse? \\
\hline & Organizado? \\
\hline & Padronizado? \\
\hline & Tamanho das letras? \\
\hline & Tamanho dos botões? \\
\hline & Navegação padronizada? \\
\hline \multirow[t]{4}{*}{ Funcionalidades } & Apresenta falha carregamento? \\
\hline & Carregamento rápido? \\
\hline & Falha (erro) durante a execução? \\
\hline & Fácil de utilizar? \\
\hline \multirow[t]{5}{*}{ Qualidade Pedagógica } & A Linguagem é adequada a idade? \\
\hline & A sequência do conteúdo é adequada? \\
\hline & A Extensão (quantidade de informação, conteúdo, duração) é adequada? \\
\hline & Possui erros de linguagem? \\
\hline & Existe alguma restrição de tempo na execução das tarefas? \\
\hline \multirow{4}{*}{$\begin{array}{ll}\text { Objetivos } & \text { de } \\
\text { Aprendizagem }\end{array}$} & Os objetivos de aprendizagem são claros? \\
\hline & Atende completamente o conteúdo ou tópico proposto? \\
\hline & Os objetivos de aprendizagem podem ser cumpridos? \\
\hline & É flexível ou permite adaptar a utilização? \\
\hline \multirow[t]{5}{*}{ Feedback } & Existe um feedback ao usuário? \\
\hline & Qual o tipo de feedback Visual, escrito, sonoro? \\
\hline & O tempo de feedback é adequado? \\
\hline & Existe ação corretiva para os erros dos usuários? \\
\hline & Propostas de atividades podem ser refeitas? \\
\hline \multirow[t]{4}{*}{ Motivação } & É visualmente atraente? \\
\hline & Propõe desafios ao usuário? \\
\hline & Está contextualizado na realidade do aluno? \\
\hline & Oferece algum tipo de punição ao erro? \\
\hline
\end{tabular}

Fonte: Elaboração própria. 


\section{Resultados e conclusões}

Depois de submetermos os 30 OA aos critérios de avaliação definidos, os dados foram organizados em tabelas para melhor compreensão e visualização dos resultados. Durante o processo podemos perceber que, de maneira geral, o instrumento proposto mostrou-se adequado para realizar uma avaliação prévia dos OA, pois grande parte das dos aspectos essenciais e necessários para o uso desses recursos foram contemplados. Diante do grande número de critérios, optamos por comentar apenas alguns resultados que evidenciam a pertinência do modelo proposto.

Entre os objetos sorteados para a amostra encontramos a seguinte composição: 8,3\% eram animações, 25\% simulações, 70,8\% Atividades de Exercício e Prática e $8,3 \%$ pequenos jogos educacionais. Aproximadamente $90 \%$ traziam figuras ou animações no design e apresentavam uma disposição adequada e equilibrada dos elementos na tela. Com relação ao uso do teclado, aspecto funcionalmente importante para as LDI, 45\% não apresentavam a necessidade de utilização do mesmo, 45\% exigiam o uso e apenas $10 \%$ exigiam o uso e apresentaram dificuldades ao utilizador. No que diz respeito ao tamanho das letras e botões nas telas, grande parte foi considerada adequada ( $87 \%$ e $91 \%$ respectivamente). Do conjunto analisado, $8 \%$ apresentou alguma falha durante a utilização e $90 \%$ foi considerado de fácil utilização.

No que diz respeito aos aspectos pedagógicos, $87 \%$ dos OA possuíam uma linguagem apropriada para a idade dos alunos e $70 \%$ foram considerados bons com relação a quantidade e qualidade das informações apresentadas sobre o tópico do currículo focalizado. Também $87 \%$ dos OA foram considerados bons no que diz respeito à sequência de abordagem dos assuntos e tópicos. Em sua grande maioria (87\%), os objetivos de aprendizagem foram considerados claros, o que permitiria consequentemente um bom aproveitamento em sala de aula. Aproximadamente 55\% dos OA possuíam características que permitiam um uso flexível em 2 ou mais temas do currículo do 3 ano do Ensino Fundamental.

Verificamos que $70 \%$ dos OA, durante sua utilização, ofereciam um feedback visual ao usuário e apenas $25 \%$ de forma escrita, esse aspecto está relacionada ao fato de que a grande maioria dos OA eram direcionados a realização de exercícios e práticas sobre um determinado tema. $75 \%$ dos recursos trazia alguma ação corretiva, no caso de erro por parte do usuário e em sua grande maioria essa ação corretiva foi considerada adequada. 
De maneira geral, este trabalho é uma iniciativa que oferece bases para uma possível metodologia para avaliação de OA para uso em Lousas Digitais, aspecto que é de fundamental importância para o êxito do uso da tecnologia em sala de aula. É importante reforçar, conforme Galvis $(1988$, p.134) aponta, que "[...] o computador deve ser usado no processo ensino aprendizagem, antes de qualquer outra coisa, como um meio para implementar o que com outros meios não seria possível ou seria difícil obter". Sendo assim, verifica-se que o bom software educacional agregado, o bom uso da tecnologia da informática na educação propiciará experiências educacionais inovadoras e enriquecedoras, ou pelo menos tornará muito mais eficiente o ensino efetivado nos moldes tradicionais.

Embora alguns modelos de avaliação de OA existentes procurem cumprir os procedimentos definidos nas Normas internacionais de qualidade, é o usuário final, professor ou aluno, aquele que fará o uso do software no dia-a-dia, quem dá o veredicto final da aceitação ou rejeição. Desta forma, as metodologias de avaliação de software adotadas devem levar em consideração o paradigma educacional que permeia o software a ser avaliado, sob pena de não atender a produção e utilização de muitos aplicativos que não estarão em conformidade com os requisitos do paradigma pedagógico adotado. Assim, consideramos viável e profícuo o modelo proposto, pois busca discutir elementos e características que assegurem uma maior probabilidade de sucesso de uso da tecnologia no âmbito educacional.

\section{LEARNING OBJECTS AND INTERACTIVE WHITEBOARDS: A EVALUATION PROPOSAL OF LEARNING OBJECTS FOR MATHEMATICS TEACHING}


Abstract: The current conditions of the classroom learning tend to be a one-way process based in teacher exposition, this make a negative impact on learning make it a mechanical and not meaningful activity. One possibility to improve the quality of teaching is to innovate methodologies and varying forms of presenting information to students, such as the use of technology in the teaching process. The Interactive Whiteboard $(I B W)$ is one of the technologies that are being implemented in Brazilian schools. One of the promising possibilities to add value to the use of LDI in classroom are "learning objects" (LO). However, one problem is that often the LO are not fully suited to the dynamics of IWB, whether functional or pedagogical point of view. The objective of this study is to analyze and propose a set of indicators that evaluate the learning objects for use in conjunction with Interactive Whiteboards. The selection and definition of evaluation indicators was carried from the literature review on the subject and based on LDI experiences of use in Municipal Elementary School. After defining the set of indicators was conducted a evaluation of a sample of 30 OA utilized to teaching mathematics in 3rd grade of elementary school. The results of the evaluation indicate that the proposed indicators are suitable for a pre-analysis of OA and assisting in the process of selection of these.

Key words: Technology. Learning objects. Digital whiteboards. Software evaluation. Indicators.

\section{REFERÊNCIAS}

ALMEIDA, R. A.; CHAVES, A. L.; COUTINHO, F. A. Avaliação da usabilidade e da qualidade do conteúdo de Objetos de Aprendizagem digitais sobre o sistema digestório. II SIMPÓSIO NACIONAL DE ENSINO DE CIÊNCIA E TECNOLOGIA, 21., 2010, Ponta Grossa. Anais... Ponta Grossa: SINECT, 2010.

ANDREWS, D. H.; GOODSON, L. A. A comparative analysis of models of instructionaldesign. Journal of Instructional Development, [S.1.], v.3, n.4, p.2-16. 1980 .

BALANSKAT, A.; BLAMIRE, R.; KEFALA, S. The ICT Impact Report: a review of studies of ICT impact on schools in Europe. [S.1.]: European Schoolnet, 2006.

Disponível em:

<http://insight.eun.org/ww/en/pub/insight/misc/specialreports/impact_study.htm>.

Acesso em: 5 nov. 2012.

BECTA. Learning in the 21st century. Coventry, UK: Becta, 2007. Disponível em: $<$ http://www.e-learningcentre.co.uk/Resource/CMS/Assets/5c10130e-6a9f-102c-a0be003005 bbceb4/form_uploads/review_early_years_foundation.pdf $>$. Acesso em: 12 mar. 2012.

BOSLEY, C.; MOON, S. Review of existing literature on the use of information and Communication Technology within an educational context. Derby: Centre for Guidance Studies, University of Derby, 2003. 
CAMPOS, G. H. B.; ROCHA, A. R. C. Manual de avaliação de software educacional. Relatório Técnico do Programa de Engenharia de Sistemas. Es-232/91. Rio de Janeiro: COPPE/UFRJ, 1991.

CASTRO-FILHO, J. A. et al. Quando objetos digitais são efetivamente para aprendizagem: o caso da matemática. In: XIX SIMPÓSIO BRASILEIRO DE INFORMÁTICA NA EDUCAÇÃO (SBIE), 1., 2008, Fortaleza. Anais... Fortaleza: Sociedade Brasileira de Computação, 2008. p.583-592.

GALVIS, A. H. Ambientes de enseñanza aprendizage enriquecidos con computador. Boletin de Informatica Educativa, Bogotá, v.1, n.2, p.117-139, dez. 1988.

GIERE, R. Explaining science. Chicago: University of Chicago Press, 1988.

GILBERT, J. K.; BOULTER, C.; RUTHERFORD, M. Models in explanations, part 1: horses for courses. International Journal of Science Education, [S.1.], v.20, n.1, p.8397, 1998.

HENNING, P. Everyday cognition and situated learning. In: JONASSEN, D. (Ed.). Handbook of Research on Educational Communications and Technology. 2.ed. New York: Simon \& Schuster, 1998. p.120-131.

KOCHANSKI, D. Um Framework para apoiar a construção de experimentos na avaliação empírica de jogos educacionais. 2009. 225 f. Dissertação (Mestrado em Computação Aplicada) - Universidade do Vale do Itajaí, São José, 2009.

OLIVEIRA, C. C. Ambientes informatizados de aprendizagem: produção e avaliação de software educativo. Campinas: Papirus, 2001.

PEDERSEN, S. G. et al. (Ed.). eLearning Nordic 2006. Copenhagen: Ramboll Management, 2006. Disponível em: <www.ramboll-management.com>. Acesso em: 02 mai. 2016.

REATEGUI, E.; FINCO, M. D. Proposta de diretrizes para Avaliação de Objetos de Aprendizagem considerando aspectos pedagógicos e técnicos. Revista Renove, Porto Alegre, v.8, n.3, p.1-10, 2010.

ROCHA, A. C.; CAMPOS, G. H. B. Avaliação da qualidade de software educacional. Órgão de Divulgação do Ministério da Educação e do Desporto. Em Aberto, Brasília, ano XII, n.57, jan./mar. 1993.

SANTOS, J. J. A. S.; MOITA, F. M. G. S. C. Objetos de aprendizagem e o ensino de matemática: análise de sua importância na aprendizagem de conceitos de probabilidade. In: II ENCONTRO REGIONAL DE EDUCAÇÃO MATEMÁTICA, 2., 2009, Natal. Anais... Natal: Universidade Federal do Rio Grande do Norte, 2009. Disponível em: <http://www.sbemrn.com.br/site/II\%20erem/comunica/doc/comunica13.pdf >. Acesso em: 11 jul. 2015.

TOMASI, J. Models and modelling in theoretical chemistry. Journal of Molecular Structure, [S.1.], v.179, p.273-292, 1988. 
UNITED STATES OF AMERICA. Office of Educational Technology. National Education Technology Plan 2010. [S.1.]: Government Report - USA, 2010. Disponível em: 〈http://www.ed.gov/technology/netp-2010〉. Acesso em: 10 mar. 2012.

VIEIRA, F. M. S. Avaliação de software educativo: reflexões para uma análise criteriosa. [Online]. 2004. Disponível em:

<http://www.edutec.net/Textos/Alia/MISC/edmagali2.htm>. Acesso em: 09 mai. 2015. 